\title{
Susceptibilidad antimicrobiana de Campylobacter jejuni aislado de coprocultivos en Santiago de Chile
}

\author{
Patricia García C., Natalia Valenzuela S., M. Victoria Rodríguez L., Eugenia León C. y Heriberto Fernández J.
}

\section{Antimicrobial susceptibility of Campylobacter jejuni isolates from stool cultures in Santiago, Chile}

Campylobacter jejuni is a common agent of enterocolitis in humans. Campylobacteriosis has been recognized as a zoonotic disease whose reservoir is the intestinal flora of poultry. The reposition of fluid and electrolytes is the recommended treatment, and antimicrobials are required only in severe and/or in prolonged disease. Given the emergence of resistance to drugs commonly used in the treatment of acute diarrhea, we studied the antimicrobial susceptibility of 73 strains of Campylobacter jejuni isolated from stool culture. The antimicrobials tested were: erythromycin, azithromycin, ampicillin and ciprofloxacin. Of the 73 strains tested by E-test, $32.4 \%$ were resistant to ciprofloxacin and $6.4 \%$ were resistant to ampicillin. Resistance to erythromycin and azithromycin was not detected. The surveillance of antimicrobial resistance of Campylobacter jejuni is important in the evaluation of empirically used antimicrobials in the treatment of bacterial enterocolitis.

Key words: Campylobacter jejuni, enterocolitis, antimicrobial susceptibility.

Palabras clave: Campylobacter jejuni, enterocolitis, susceptibilidad antimicrobiana.

\section{Introducción}

C ampylobacter jejuni fue identificado como patógeno humano intestinal por primera vez en 1972. Campylobacter es un bacilo gramnegativo curvo, con forma característica de "sacacorchos", cuya motilidad está dada por un flagelo polar presente en uno o ambos extremos. Las especies Campylobacter jejuni y Campylobacter coli son microaerofílicos y termotolerantes (crecen a $42^{\circ} \mathrm{C}$ ); estaúltima característica permite su diferenciación con Campylobacter fetus ${ }^{1-4}$. Ambas especies han sido reconocidas como los patógenos humanos más importantes de este género bacteriano y son agentes habituales de enterocolitis ${ }^{5}$.

La infección por Campylobacter en humanos ha sido catalogada como una zoonosis ${ }^{2,3,5-7}$, teniendo como reservorio más importante las aves de corral, donde forma parte de la microbiota intestinal ${ }^{3,6,8}$.

Campylobacter es un agente común de diarrea en el mundo. Según reportes del CDC, durante 1996, 46\% de las gastroenteritis bacterianas comprobadas por cultivo en E.U.A. tuvieron como agente etiológico a Campylobacter $\mathrm{sp}^{2}$. También se ha reportado que en países desarrollados, el número de casos causados por este agente excede el total de los casos causados por Salmonella sp, Shigella sp y Escherichia coli 0157: H7.
En nuestro país, en estudios realizados hace 20 años en las ciudades de Concepción, Valparaíso y Santiago, este agente ocupaba el tercer lugar entre las diarreas agudas de causa bacteriana en niños bajo dos años de edad ${ }^{9-11}$. Chanqueo L y cols, en el año 2005, reportaron Campylobacter sp como el segundo enteropatógeno aislado con mayor frecuencia en muestras de coprocultivo de todos los rangos etáreos ${ }^{12}$.

Con respecto al tratamiento, la reposición de fluidos y electrolitos es lo recomendado y solamente requieren antimicrobianos los casos graves y/o prolongados. Los fármacos de elección para estos casos han sido por largo tiempo los macrólidos, azólidos y fluoroquinolonas como ciprofloxacina. Sin embargo, existen diversos reportes sobre la aparición de resistencia a fluroquinolonas y tetraciclina a nivel mundial ${ }^{2,5-13-22}$. Se ha descrito un bajo nivel de resistencia para eritromicina, el fármaco de elección, mientras que para azitromicina se ha reportado resistencia hasta en 31\% de las cepas aisladas en países asiáticos². Considerando estos antecedentes, la Organización Mundial de la Salud no recomienda tratar en forma empírica una campilobacteriosis sin una previa evaluación de la susceptibilidad in vitro ${ }^{2}$.

A pesar de las recomendaciones existentes, en nuestro país no se realiza la vigilancia epidemiológica de resistencia antimicrobiana ya que por los requerimientos de
Pontificia Universidad Católica de Chile, Santiago Escuela de Medicina Departamento de Laboratorios Clínicos (PGC, NVS) Servicio de Laboratorio Clínicos (MVRL)

Laboratorio de Microbiología (ELC) Universidad Austral de Chile, Valdivia

Instituto de Microbiología Clínica (HFJ)

Los autores declaran no tener conflictos de

interés involucrados en este trabajo

Proyecto financiado por el Departamento de Laboratorios Clínicos de la Pontificia Universidad Católica de Chile

Recibido: 7 de julio de 2009 Aceptado: 3 de octubre de 2009

Correspondencia a: Patricia García Cañete pgarcia@med.puc.cl 
Campylobacter para su crecimiento, los laboratorios de microbiología no efectúan la búsqueda rutinaria de este agente con la consiguiente subvaloración de su importancia epidemiológica ${ }^{7}$. Dada la progresiva resistencia a quinolonas reportada en el mundo ${ }^{13-16,19-22}$, el objetivo de nuestro trabajo fue conocer la susceptibilidad antimicrobiana de $C$. jejuni a los fármacos utilizados de forma empírica en el tratamiento de la diarrea aguda.

\section{Material y Método}

Cepas bacterianas. Las cepas fueron obtenidas en forma prospectiva a partir de muestras de deposiciones recibidas para coprocultivo en el Laboratorio de Micro-

\section{Tabla 1. Puntos de corte para los antimicrobianos ensayados}

\begin{tabular}{lcccc|} 
Antimicrobiano & Referencia & $\begin{array}{c}\text { Susceptible } \\
(\boldsymbol{\mu} \mathbf{g} / \mathbf{m L})\end{array}$ & $\begin{array}{c}\text { Intermedio } \\
(\boldsymbol{\mu} \mathbf{g} / \mathbf{m L})\end{array}$ & $\begin{array}{c}\text { Resistente } \\
(\boldsymbol{\mu} \mathbf{g} / \mathbf{m L})\end{array}$ \\
Ampicilina & 25 & $\leq 8$ & 16 & $\geq 32$ \\
Eritromicina & 24 & $\leq 8$ & 16 & $\geq 32$ \\
Azitromicina & 26 & $\leq 4$ & - & $\geq 8$ \\
Ciprofloxacina & 24 & $\leq 1$ & 2 & $\geq 4$ \\
\hline
\end{tabular}

Tabla 2. Susceptibilidad antimicrobiana de cepas de Campylobacter jejuni aisladas en la ciudad de Santiago de Chile $(n=73)$

\begin{tabular}{lccc|} 
Antimicrobiano & Sensible (\%) & Intermedio (\%) & Resistente (\%) \\
\hline Ampicilina & $69(93,2)$ & $4(5,4)$ & $1(1,4)$ \\
Eritromicina & $73(100)$ & 0 & 0 \\
Azitromicina & $73(100)$ & 0 & 0 \\
Ciprofloxacina & $50(67,6)$ & 0 & $24(32,4)$ \\
\hline
\end{tabular}

Tabla 3. Concentración inhibitoria mínima 50 y $90^{*}$ para los antimicrobianos ensayados

\begin{tabular}{|c|c|c|}
\hline Antimicrobiano & $\begin{array}{c}\mathrm{CIM}_{50} \\
(\mu \mathrm{g} / \mathrm{mL})\end{array}$ & $\begin{array}{c}\mathrm{CIM}_{90} \\
(\mu \mathrm{g} / \mathrm{mL})\end{array}$ \\
\hline Ampicilina & 2 & 8 \\
\hline Eritromicina & 0,5 & 2 \\
\hline Azitromicina & 0,125 & 1 \\
\hline \multicolumn{3}{|c|}{$\begin{array}{l}{ }^{*} \mathrm{CIM}=\text { Concentración inhibitoria mínima. } \mathrm{CIM}_{50}=\text { Aquella concentración de antimicrobiano que } \\
\text { inhibe el desarrollo del } 50 \% \text { de las cepas estudiadas. } \mathrm{CIM}_{90}=\text { Aquella concentración de antimicrobiano } \\
\text { que inhibe el desarrollo del } 90 \% \text { de las cepas estudiadas }\end{array}$} \\
\hline
\end{tabular}

biología de la Red de Salud de la Pontificia Universidad Católica de Chile, entre los años 2002 y 2007 en la ciudad de Santiago. Las muestras fueron sembradas en agar selectivo para Campylobacter e incubadas en microaerofilia a $42^{\circ} \mathrm{C}$ por 48 horas. La identificación de Campylobacter jejuni se realizó por las características de la colonia, características tintoriales (tinción de Hucker) e hidrólisis de hipurato positiva. Una vez identificadas, las cepas se almacenaron a $-80^{\circ} \mathrm{C}$ en caldo glicerol hasta el momento del estudio.

Estudio de susceptibilidad in vitro. Se realizaron dos resiembras de las cepas congeladas, una por 48 horas y la segunda por 24 horas, ambas en agar sangre y en microaerofilia. Posteriormente, se realizó una suspensión bacteriana con un patrón de turbidez equivalente al estándar MacFarland 1,0 en caldo Mueller-Hinton ${ }^{23}$. De esta suspensión se dispensaron $100 \mu \mathrm{L}$ en una placa de agar Mueller Hinton con sangre de cordero al 5\% sembrando por inundación. El estudio de susceptibilidad se realizó por epsilometría (E-test $\AA$, AB Biodisk, Suecia). Los antimicrobianos evaluados fueron eritromicina, ensayándose concentraciones entre 0,016 y $256 \mu \mathrm{g} / \mathrm{mL}$, azitromicina, ensayándose concentraciones entre 0,016 y $25 \mu \mathrm{g} / \mathrm{mL}$, ampicilina, ensayándose concentraciones entre 0,016 y $256 \mu \mathrm{g} / \mathrm{mL}$ y ciprofloxacina, ensayándose concentraciones entre 0,002 y $32 \mu \mathrm{g} / \mathrm{mL}$. Las placas fueron incubadas por 48 horas a $35^{\circ} \mathrm{C}$ en microaerofilia. La interpretación de los puntos de corte se realizó según los criterios establecidos por $\mathrm{CLSI}^{24,25}$ (Tabla 1). Para azitromicina se utilizaron los puntos de corte establecidos por National Antimicrobial Resistance Monitoring System (NARMS) en que se considera susceptible una cepa con una concentración inhibitoria mínima $(\mathrm{CIM}) \leq 4 \mu \mathrm{g} / \mathrm{mL}$ y resistente $\geq 8 \mu \mathrm{g} / \mathrm{mL}$ ya que no existen puntos de corte determinados por $\mathrm{CLSI}^{26}$. Como control se utilizó la cepa C. jejuni ATCC 33560.

\section{Resultados}

Durante el período de estudio se recolectaron 73 cepas de $C$. jejuni aisladas de coprocultivos. El promedio de edad de los pacientes evaluados fue de 8,9 años (con un rango de edad de 6 meses a 68 años, con una mediana de 3,8 años y una moda de 1,8 años). El estudio de susceptibilidad demostró $24(32,4 \%)$ cepas resistentes a ciprofloxacina (CIM $>32 \mu \mathrm{g} / \mathrm{mL}$ ) y una a ampicilina $(\mathrm{CIM}>256 \mu \mathrm{g} / \mathrm{mL})$. El 5,4\% (4/73) de las cepas presentó resistencia intermedia a ampicilina. Ninguna de las cepas evaluadas presentó resistencia a eritromicina y azitromicina ni a más de un antimicrobiano (Tabla 2). Las CIM 50 y 90 se describen para cada antimicrobiano en la Tabla 3. 


\section{Discusión}

Campylobacter jejuni es un agente importante de enterocolitis bacteriana en el mundo, especialmente en pacientes en edad pediátrica ${ }^{9,27}$. A pesar de que el tratamiento antimicrobiano no es indicación de rutina, en los últimos años se ha hecho importante conocer su susceptibilidad dada la alta tasa de resistencia reportada en la literatura médica² ${ }^{2}$.

Los datos obtenidos en este estudio demuestran que nuestro país no es ajeno a esa realidad, ya que $32,4 \%$ de las cepas aisladas en nuestro laboratorio fueron resistentes a ciprofloxacina. Esta cifra representa un cambio en el patrón de resistencia si consideramos que, en aislados realizados entre los años 1996 y 1997 en el sur de Chile, Fernández y cols, reportaron $100 \%$ de susceptibilidad a ciprofloxacina $^{18}$, aunque la presencia de este tipo de resistencia ya había sido detectada en $1996^{27}$. Las quinolonas tienen dos sitios blanco en las bacterias gramnegativas: un sitio principal (ADN girasa o topoisomerasa II) y un sitio secundario (topoisomerasa IV). En Campylobacter, el blanco secundario está ausente, lo que está dado por una mutación puntual que determina un cambio aminoacídico (Thr-86 a Ile) en la región blanco de la topoisomerasa II, denominada "región determinante de resistencia a las quinolonas" (RDRQ). La no existencia del blanco secundario permite que esta modificación sea suficiente para que Campylobacter presente resistencia de alto nivel a fluoroquinolonas $(\mathrm{CIM} \geq 16 \mu \mathrm{g} / \mathrm{mL})$. Esta mutación no confiere resistencia a otras quinolonas más complejas; sin embargo, se ha descrito que una doble mutación (Thr86Ile-Asp90Asn) es capaz de producir resistencia a moxifloxacina ${ }^{28}$.

Es importante destacar que, al igual que lo reportado por otras series, la resistencia a ciprofloxacina está presente en cepas aisladas principalmente de pacientes pediátricos en los cuales no se utilizan quinolonas como tratamiento empírico de diarrea. Dada esta situación, es válido suponer que la probabilidad de que este tipo de resistencia haya sido adquirida "intra-tratamiento" es baja, pudiendo asumir que la infección ha sido provocada por bacterias previamente resistentes ${ }^{13-14}$. Diversas publicaciones han asociado el aumento de la resistencia de Campylobacter a las quinolonas con la introducción de estos fármacos en la industria avícola, principalmente enrofloxacina, cuyo metabolito activo es ciprofloxacina ${ }^{17,21,29,30}$. A pesar de que en nuestro país se realiza una vigilancia de los niveles permitidos de este medicamento en la industria avícola, no existen publicaciones sobre la resistencia de cepas de Campylobacter aisladas de aves para consumo humano.

Con respecto a los otros antimicrobianos evaluados, no se observó resistencia a macrólidos ni azólidos, que son los antimicrobianos de elección. En cambio, nuestras cepas presentaron $6,8 \%$ de resistencia a ampicilina, cifra similar a la descrita por Fernández y cols ${ }^{18}$. Campylobacter es intrínsecamente resistente a penicilina $G$ y cefalosporinas de primera generación debido a la débil unión de estos antimicrobianos con las proteínas de unión a penicilina (PBPs) ${ }^{31}$. Además, se ha descrito la adquisición de $\beta$-lactamasas como una causa importante de resistencia a $\beta$-lactámicos en esta bacteria ${ }^{31-33}$. En un estudio realizado por Tajada y cols, se determinó que de 100 cepas de C. jejuni y Campylobacter coli, 88\% presentaban $\beta$-lactamasas y que, pese a la existencia de éstas, $77 \%$ de las cepas que las poseían eran susceptibles a amoxicilina ${ }^{34}$. Diversos autores plantean que esta situación se debe a la presencia de porinas en la pared de Campylobacter que facilitarían el ingreso de moléculas como imipenem, ampicilina, amoxicilina y cefpirome permitiendo la acción de estos agentes a pesar de la presencia de $\beta$-lactamasas en la bacteria ${ }^{32-35}$. A pesar de que ampicilina no es un fármaco de utilidad en el tratamiento de la infección por Campylobacter ${ }^{7}$ se ha descrito 14 a $40 \%$ de resistencia a este antimicrobiano, lo que hace razonable la monitorización de su susceptibilidad in vitro con fines epidemiológicos ${ }^{35}$.

Dado que el patrón de resistencia antimicrobiana de C. jejuni ha presentado variaciones en los últimos años, creemos que es importante realizar la vigilancia de su susceptibilidad in vitro, especialmente para aquellos antimicrobianos utilizados de manera empírica en el tratamiento de la enterocolitis bacteriana y, especialmente, en aquellas cepas aisladas de pacientes con enfermedades invasoras o inmunocomprometidos.

\section{Resumen}

Campylobacter jejuni es un agente habitual de enterocolitis en humanos. La campilobacteriosis ha sido catalogada como una zoonosis cuyo reservorio es la microbiota intestinal de las aves de corral. El tratamiento con antimicrobianos sólo está recomendado a los casos graves y/o prolongados. Con el fin de evaluar la susceptibilidad in vitro de Campylobacter jejuni, dado la aparición de resistencia a los fármacos utilizados habitualmente en el tratamiento de la diarrea aguda, se realizó este estudio en 73 cepas aisladas de coprocultivo. Los antimicrobianos evaluados fueron: eritromicina, azitromicina, ampicilina y ciprofloxacina. De las 73 cepas evaluadas por epsilometría, 32,4\% de las cepas fue resistente a ciprofloxacina y $6,4 \%$ presentó susceptibilidad dismunuida a ampicilina. No se detectó resistencia a eritromicina ni azitromicina. Es importante realizar la vigilancia de resistencia antimicrobiana en $C$. jejuni para conocer su patrón de susceptibilidad y evaluar aquellos antimicrobianos utilizados empíricamente en el tratamiento de la enterocolitis bacteriana. 


\section{Referencias}

1.- Nachamkin I. Campylobacter and Arcobacter. Murray P, Baron E, Pfaller M, Jorgesen J, Yolken R, editors. Manual of Clinical Microbiology. $9^{\text {th }}$ edition. Washington D.C.ASM Press, 2007; p. 933-46.

2.- Sack D, Lyke C, McLaughlin C, Suwanvanichkij V. Antimicrobial resistance in shigellosis, cholera and campylobacteriosis. WHO/CDS/DRS/2001.8.

3.- Park S. The physiology of Campylobacter species and its relevance to their role as foodborne pathogens. Int J Food Microbiol 2002; 74: 177-88

4.- $\quad$ Young K, Davis L, DiRita V. Campylobacter jejuni: molecular biology and pathogenesis. Nat Rev Microbiol 2007; 5: 665-79.

5.- Peterson M. Clinical aspects of Campylobacter jejuni infections in adults. West J Med 1994; 161: 148-52.

6.- Newell D, Feanley C. Sources of Campylobacter colonization in broiler chickens. Appl Environ Microbiol 2003; 69: 4343-51.

7.- Blaser M. Epidemiologic and clinical features of Campylobacter jejuni infections. J Infect Dis 1997; 176: S103-5.

8.- Figueroa A, Ruiz M, Adriazola P, Figueroa G. Prevalencia de Campylobacter jejuni em carnes de ave. Libro de resúmenes XXVI Congreso Chileno de Microbiologia. Valparaíso, Chile; 2004; p. 215.

9.- Henríquez M, Venegas G, Soto G, Rudolff T, Salas J. Etiología bacteriana de la diarrea aguda del lactante en otoño e invierno. Rev Chil Pediatr 1985; 56: 450-3.

10.- Prado V. Epidemiología de la enteritis por Campylobacter en niños. Rev Méd Chile 1984; 112: 1153.

11.- Prado V, Martínez J, Reyes L, Ducheylard M, Bercovich M, Millán V, et al. Características de la infección intestinal por Campylobacter jejuni en lactantes chilenos. Rev Méd Chile 1985; 113: 521-5.

12.- Chanqueo L, García P, León E, Blu A. Evaluación de la tinción de Hucker para la búsqueda rutinaria de Campylobacter sp en el estudio de un síndrome diarreico agudo. Rev Chil Infect 2005; 22: 242-46

13.- Butzler J. Campylobacter, from obscurity to celebrity. Clin Microbiol Infect 2004; 10: 868676.

14.- Molina J, Casin I, Hausfater P, Giretti E, Welker Y, Decazes J, et al. Campylobacter infections in HIV-infected patients: clinical and bacteriological features. AIDS 1995; 9: 881-5.

15.- Sánchez R, Fernández-Baca V, Díaz M, Muñoz P, Rodríguez-Creixems M, Bouza E. Evolution of susceptibilities of Campylobacter spp to quinolones and macrolides. Antimicrob Agents Chemother 1994; 38: 1879-82.

16.- Schonberg-Norio D, Hanninen M, Katila M, Kaukoranta S, Koskela M, Eerola E, et al. Activities of telithromycin, erythromycin, fluroquinolones and doxycyline against Campylobacter strains isolated from finnish subjects. Antimicrob Agents Chemother 2006; 50: 1086-8.

17.- Vanhoof R, Gossens H, Coignau H, Stas G, Butzler J. Susceptibility pattern of Campylobacter jejuni from human and animal origins to different antimicrobial agents. Antimicrob Agents Chemother 1982; 21: 990-2.

18.- Fernández H, Mansilla M, González V. Antimicrobial susceptibility of Campylobacter jejuni subsp. jejuni assessed by E-test and double dilution agar method in Southern Chile. Mem Inst Oswaldo Cruz. 200; 95: 247-9.

19.- Senok A, Yousif A, Mazi W, Sharif E, Bindayana K, Elnima E. Pattern of antibiotic susceptibility in Campylobacter jejuni isolates of human and poultry origin. Jpn J Infect Dis 2007; 60: 1-4.

20.- Sanders J W, Isenbarger D, Walz S, Pang L, Scott D, Tamminga C, et al. An observational clinic-based study of diarrheal illness in deployed United States military personnel in Thailand: presentation and outcome of Campylobacter infection. Am J Trop Med Hyg 2002; 67: 533-8.

21.- Wardak S, Szych J, Zasada A, Gierczynski R. Antibiotic resistance of Campylobacter jejuni and Campylobacter coli clinical isolates from Poland. Antimicrob Agents Chemother 2007; 51: 1123-25.

22.- Cardinale E, Dromigny JA, Tall F, Ndiaye M, Konte M, Perrie-Gros-Claude J. Fluoroquinolone susceptibility of Campylobacter strains, Senegal. Emerg Infect Dis 2003; 9: 1479-81.

23.- Luber P, Bartelt E, Genschow E, Wagner J, Hahn H. Comparison of broth microdilution, E-test, and agar dilution methods for antibiotic susceptibility testing of Campylobacter jejuni and Campylobacter coli. J Clin Microbiol 2003; 41: 1062-8.

24.- Clinical and Laboratory Standards Institute. Methods for antimicrobial dilution and disk susceptibility testing of infrequently isolated or fastidious bacteria: proposed guideline. CLSI document M45-P Wayne, Pennsylvania: Clinical and Laboratory Standards Institute; 2005, p. 16, 39.

25.- Clinical and Laboratory Standards Institute. Performance standards for antimicrobial susceptibility testing; seventeenth informational supplement. CLSI document M100-S17. Wayne, Pennsylvania: Clinical and Laboratory Standards Institute; 2007, p. 130-32.

26.- National antimicrobial resistance monitoring system for enteric bacteria (NARMS): Human isolates. Final Report. Atlanta, Georgia: US Department Of Health and Human Services, CDC; 2006, p56-61.

27.- Fernández H, Martin R, Thibaut J. Campylobacter jejuni y Campylobacter coli resistentes a fluoroquinolonas en animales domésticos. Arch Med Vet 1996; 28: 151-4.

28.- Payot S, Bolla J, Corcoran D, Fanning S, Mégraud F, Zhang Q. Mechanism of fluoroquinolone and macrolide resistance in Campylobacter spp. Microbes Infect 2006; 8: 1967-72.

29.- Alfredson D, Akhurst R, Korolik V. Antimicrobial resistance and genomic screening of clinical isolates of thermophilic Campylobacter spp. from South-East Queensland, Australia. J Appl Microbiol 2003; 94: 495-500.

30.- Threlfall E. Fluoroquinolone-resistant Campylobacter and animal drug use. Int J Infect Dis 2004; 8: 190-2.

31.- Lachance N, Gaudreau C, Lamothe F, Larivière L. Role of the $\beta$-lactamase of Campylobacter jejuni in resistance to $\beta$-lactamase agents. Antimicrob Agents Chemother 1991; 35: 813-18.

32.- Gaudreau C, Gilbert H. Comparison of disc diffusion and agar dilution methods for antibiotic susceptibility testing of Campylobacter jejuni subsp. jejuni and Campylobacter coli. J Antimicrob Chemother 1997; 39: 707-12.

33.- Reina J, Ros M, Serra A. Susceptibilities to 10 antimicrobial agents of 1220 Campylobacter strains isolated from 1987 to 1993 from feces of pediatric patients. Antimicrob Agents Chemother 1994; 38: 2917-20.

34.- Tajada P, Gómez-Garcés J, Alós J, Balas D, Cogollos R. Antimicrobial susceptibilities of Campylobacter jejuni and Campylobacter coli to $12 \beta$-lactam agents and combinations with $\beta$-lactamase inhibitors. Antimicrob Agents Chemother 1996; 40: 1924-25.

35.- Gaudreau C, Gilbert H. Comparison of disc diffusion and agar dilution methods for antibiotic susceptibility testing of Campylobacter jejuni subsp. jejuni and Campylobacter coli. J Antimicrob Chemother 1997; 39: 707-12. 